\title{
Multi-agent agro-economic simulation of irrigation water demand with climate services for climate change adaptation
}

\author{
Stefano Balbi, ${ }^{1,2}$ Sabindra Bhandari, 2,3 Animesh K. Gain,, 2,4 Carlo Giupponi ${ }^{2,4}$ \\ ${ }^{1}$ Basque Centre for Climate Change (BC3), Bilbao, Spain; ${ }^{2}$ Department of Economics, Ca' Foscari \\ University of Venice, Italy; ${ }^{3}$ Center For Sustainable Development Solutions, Lalitpur, Nepal; \\ ${ }^{4}$ Euro-Mediterranean Centre for Climate Change, Island of San Giorgio Maggiore, Venice, Italy
}

\begin{abstract}
Farmers' irrigation practices play a crucial role in the sustainability of crop production and water consumption, and in the way they deal with the current and future effects of climate change. In this study, a system dynamic multi-agent model adopting the soil water balance provided by the Food and Agriculture Organization (FA0) Irrigation and Drainage Paper 56 was developed to explore how farmers' decision making may affect future water needs and use with a focus on the role of climate services, i.e. forecasts and insurance. A climatic projection record representing the down-scaled A1B market scenario (a balance across all sources) of the assessment report of the Intergovernmental Panel on Climate Change (IPCC) is used to produce future daily data about relative humidity, precipitation, temper-
\end{abstract}

Correspondence: Stefano Balbi, Basque Centre for Climate Change (BC3), Alameda Urquijo $4,4^{\circ}$, Bilbao, Spain.

E-mail: stefano.balbi@bc3research.org

Key words: micro-simulation, climate services, farmer behaviour, irrigation water demand.

Acknowledgements: the Authors wish to acknowledge the support from the Euro-Mediterranean Centre for Climate Change (CMCC) and in particular from Silvio Gualdi and Edoardo Bucchignani for climatic simulations.

Funding: this research has been partially funded by the ICARUS project (http//www.cmcc.it/research/research-projects/icarus-1) with the financial support of the Italian Institute for Environmental Protection and Research (ISPRA).

Contributions: SB carried out the development of the UML conceptual model; CG directed the research and designed the system dynamic model structure with SB; AKG contributed to manuscript writing and documentation. All the authors were involved in the simulation and testing of the prototype.

Received for publication: 9 May 2013.

Revision received: 19 July 2013.

Accepted for publication: 19 July 2013.

(C) Copyright S. Balbi et al., 2013

Licensee PAGEPress, Italy

Italian Journal of Agronomy 2013; 8:e23

doi:10.4081/ija.2013.e23

This article is distributed under the terms of the Creative Commons Attribution Noncommercial License (by-nc 3.0) which permits any noncommercial use, distribution, and reproduction in any medium, provided the original author(s) and source are credited. ature and wind speed. Two types of meteorological services are made available: i) a bi-weekly bulletin; and ii) seasonal forecasts. The precision of these services was altered to represent different conditions, from perfect knowledge to poor forecasts. Using the available forecasts, farming agents take adaptation decisions concerning crop allocation and irrigation management on the basis of their own risk attitudes. Farmers' attitudes are characterized by fuzzy classifications depending on age, relative income and crop profitability. Farming agents' adaptation decisions directly affect the crop and irrigation parameters, which in turn affect future water needs on a territorial level. By incorporating available and future meteorological services, the model allows the farmer's decision making-process to be explored together with the consequent future irrigation water demand for the period 2015 to 2030 . The model prototype is applied to a data set of the Venice Lagoon Watershed, an area of $2038 \mathrm{~km}^{2}$ in north-east Italy, for a preliminary test of its performance and to design future development objectives.

\section{Introduction}

Climate change signals are already evident in many parts of the world and significant impacts are expected in the future on agricultural systems, thus presenting a growing challenge for tactical (day to day) and strategic (medium- to long-term, i.e. from annual to multiannual) farm management.

Farmers' decision-making becomes more and more challenging, particularly where the quantity and quality of available water are at risk. Irrigation planning and management are, therefore, a crucial decision-making task for farmers in many parts of the world for agricultural productivity and sustainable economic return.

When planning and management activities are brought into the climate change perspective we speak in terms of planned (by institutions, regulations, incentives, etc.) or autonomous (by individuals) adaptation. In the agricultural sector, autonomous adaptation by farmers forms a baseline from which the need for planned anticipatory adaptation can be evaluated by policy makers (Smit and Pilifosova, 2001). Moreover, it is only with adequate insight into the ongoing and the likely future autonomous adaptation processes that planned adaptation strategies can be effective, produce the expected outcomes, and avoid possible cases of maladaptation.

Autonomous adaptation of farmers could play a crucial role as far as water saving and farm-income protection strategies are concerned. In particular, it is essential to look at how reactive private actions, some of which are already being implemented (Bojovic et al., 2012), could further contribute in the near future to the process of adaptation to climate change, and how public interventions (e.g. public investments in water infrastructures) can increase their efficiency at a minimum cost. 
Engineering and technological solutions are not the only opportunities available for climate change adaptation (CCA) as information systems are expected to play a bigger role. In accordance with Dutton (2002), we assume that, in the near future, climate services could provide a reliable tool to help decision makers in the allocation of resources through anticipation of poor, fair or good seasons, even at the middle latitudes. Accordingly, climate services could support autonomous adaptation strategies by affecting farmers' attitude and response towards crop allocation, cropping and irrigation system, insurance and other risk management strategies, and related decisions. Micro-simulation is, therefore, needed in order to investigate such dynamics on the ground and observe their outcome (Balbi et al., 2013). In particular, an agent-based model can offer an exciting opportunity to model heterogeneous agro-economic behavior, taking the farmers' viewpoint as starting point (Berger, 2001), under the effects of exogenous drivers, such as policy, climate and the market. Previous works of this kind have explored how the interaction between meteorological services and farmers' behaviour can affect crop yields and, ultimately, food security on South African subsistence farmland (Bharwani et al., 2005; Ziervogel et al., 2005). In this study, we focus on decision making about efficient water use and farm-income protection of an intensive agricultural landscape subject to impacts of climate change.

A prototype tool, based on the soil-water balance procedure by Allen et al. (1998), included in the FAO Irrigation and Drainage Paper 56, was developed in Simile (http://www.simulistics.com/), an object-oriented simulation software originally developed to represent processbased system dynamics (Bhandari, 2011). By incorporating farming agents, we modelled an agentised version of the FA0-56 procedure that enables us to represent a complex social-ecological system made up of multiple farms in a given agro-ecological environment, and investigate how farmer's decisions can affect future irrigation water demand.

We provide the conceptual model description according to the ODD+D protocol by Müller et al. (2013) which focuses on the conceptual model (overview and design concepts) with the support of a selection of unified modelling language (UML) diagrams. We also provide details about the implemented model and the preliminary model results, and we discuss the pros and cons of this simulation-based approach. Finally, conclusions are drawn about this modelling exercise and directions for consolidating use of the tool and further developments are identified.

\section{Materials and methods}

\section{Theoretical and empirical background}

The impacts of climate change on agriculture are also expected to involve the middle latitudes. Different effects may develop that alter both biotic and abiotic variables affecting yields, such as alterations on crop phenology, potential pest damage, and bio-geographical cycles. This paper focuses on climate change and water resources.

Farmers' attitudes and response to irrigation will, therefore, play an increasing role in the sustainability of crop production and water withdrawal in competition with other sectors. Innovative adaptive approaches may require substantial private and public investment (Smit and Skinner, 2002). So it is interesting to investigate the degree of autonomous adaptive capacity of agricultural systems given: i) the irrigation infrastructure in place; ii) the available meteorological services in place; iii) the role of available risk management instruments, including insurance of crop yields.
The capability of projecting likely reactions of the agricultural social-ecological systems to drivers of global change could then throw some light on how planned adaptation can be synergic with expected autonomous adaptation, for example, by investing in new infrastructure and/or increasing the quality of climate services, and/or supporting the insurance sector.

The Venice Lagoon Watershed (VLW) was selected to test the prototype; well-established databases are available (Giupponi et al., 2012) and previous studies have examined local climate change scenarios (Cossarini et al., 2008; Salon et al., 2008). Currently, the water infrastructure in the VLW is mostly based on open canals, and most of the farmers use sprinkler irrigation systems (INEA, 2009). Accordingly, different configurations are possible in the model to analyse heterogeneous conditions in irrigation (which in the current version of the model are treated as exogenous scenarios) while taking into consideration the fact that certain irrigation systems are more suitable for specific water infrastructures.

\section{Entities, state variables, and scales}

As stated above, the purpose of the model is to investigate how farmers' decisions may affect future irrigation water demand, together with their own income, under the pressure of climate change, and to study what the role of climatic services might be in coping with a possible exacerbation of problems of water scarcity.

The UML was adopted to develop and share the conceptual model within our research group and to provide a guideline for implementation in the system dynamic software. The model consists of eight main entities including: farmer, water infrastructure system, irrigation system, pixel of landscape, soil, crop, market and insurance, and climate. Furthermore, the Agri-Landscape is an aggregation of all the pixels representing the study area, while the FA0 56 Water Balance Model is a sub-model for calculating the soil water balance of each pixel. All the entities are presented in Figure 1.

Farmers are described as decision agents. The given risk attitudes of farmers affect the irrigation and crop management decisions. Farmers' risk-taking attitude is inversely related in proportion to their age and the share of their income coming from farming (Moscardi and de Janvry, 1977). The water infrastructure system is represented by the provision typology and the related system efficiency. Two types of provisions are available: i) pressurised system; and ii) open canal. The irrigation system is characterised by type and related field efficiency. For the VLW, three types are considered: i) gravitational; ii) sprinkler; and iii) drip irrigation.

The conceptual model envisages the possibility of considering agroecological zones within the Agri-Landscape on a pixel-by-pixel basis, in order to allow for future integration within a geographical information system (GIS). Every pixel represents a single farmer owning a unitary surface of Utilised Agricultural Area (UAA), characterised by a specific soil and climate, and which may be allocated to different crops. The soil entity is characterised by type, field capacity, depletion, total available water, soil water content and runoff.

Two types of crops are considered in the prototype: winter wheat and maize. The first is chosen to represent rainfed crops with cycles from autumn to late spring with limited climate sensitivity, while the latter represents the irrigated crop with a spring to autumn cycle and high water consumption and climate sensitivity.

The crop market is described in terms of crop prices and production costs, while the insurance market defines the maximum crop yield that can be covered by a multiple-peril crop (MPC) type of insurance (Iturrioz, 2009). This is based on a portion of the producer's expected yield. In order to be prudent, the expected yield is assumed to be $50 \%$.

Climate represents climatic stations characterised in terms of daily precipitation, evapotranspiration, wind speed and relative humidity, 
provided by regional climate models. The model runs with daily time steps over a period of 16 years (2015-2030), utilising a synthetic climate record derived from downscaling of the Intergovernmental Panel on Climate Change (IPCC) A1B scenario (Scoccimarro et al., 2011).

\section{Process overview and scheduling}

The model process is divided into two levels: tactical and strategic. The tactical level includes those operations that are carried out on a daily basis. The strategic level includes those operations that take place only at certain times of the year (Figure 2).

Tactical operations are related to watering and updating of the climatic data and the water balance model at the pixel level (i.e. getClimateBulletin, watering operations). The farmer provides irrigation to the field depending on: i) the crop allocation in place; ii) the average efficiency of the irrigation system in place; and iii) the available bi-weekly bulletin providing precipitation forecasts.

Strategic operations take place at very precise moments of the year. In order to allow for the crop choice between maize and wheat in a unique time interval, both the release of the forecast and the farmers' decisions about land allocation are set in mid-October. At the same time, the market computes the market fundamentals. Selling price and cost of production parameters are based on values over the year 2011 (ISMEA, 2012). It is assumed that maize can, on average, provide higher revenues per unit of UAA, but both the selling price and the cost of production are more volatile with respect to winter wheat that offers more stable revenues. The seasonal forecast contains information about the variation in expected summer precipitation compared with average values to calculate an index of drought probability. The proportion of UAA allocated to wheat is proportional to the drought index calculated for the coming season, but it depends on the risk attitude of each farmer.

Crop management follows specific sowing and harvesting schedules. Maize is sown in April and harvested in October, while winter wheat is sown in October and harvested in June. After harvesting, farmers can, to a certain extent, predict their performance in terms of crop productivity, income and water use. All the pixel values are cumulated across the whole agricultural landscape [The current version of the prototype is not spatially explicit because, even if it can manage the diversity of variable values (e.g. a cohort of farmers with different characteristics), the values are not allocated to specific spatial co-ordi-

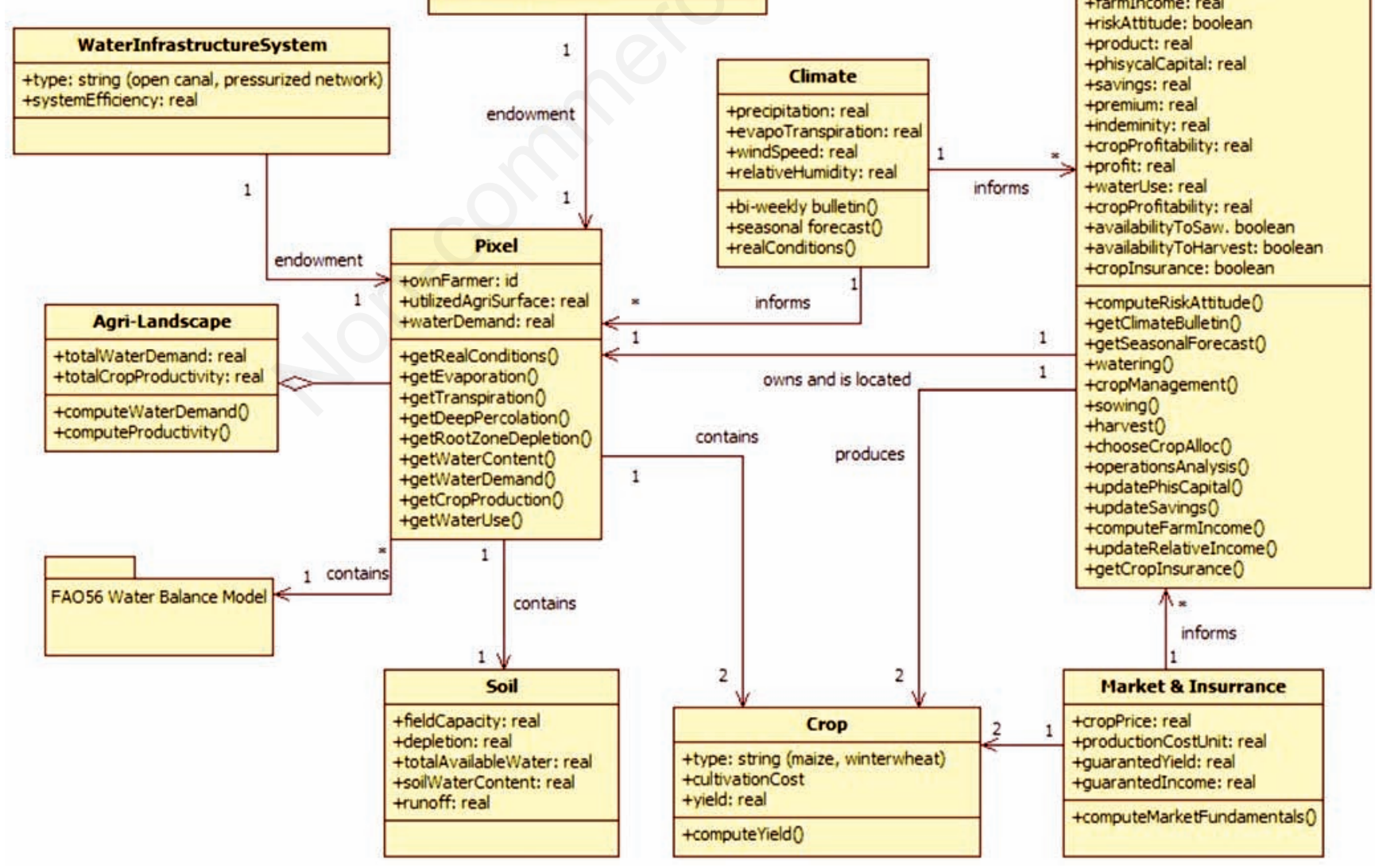

Figure 1. The unified modelling language class diagram showing the name of each model entity (top of box), its state variables (middle), and its operations (bottom). Each entity may relate with others according to different correspondences of instances. 


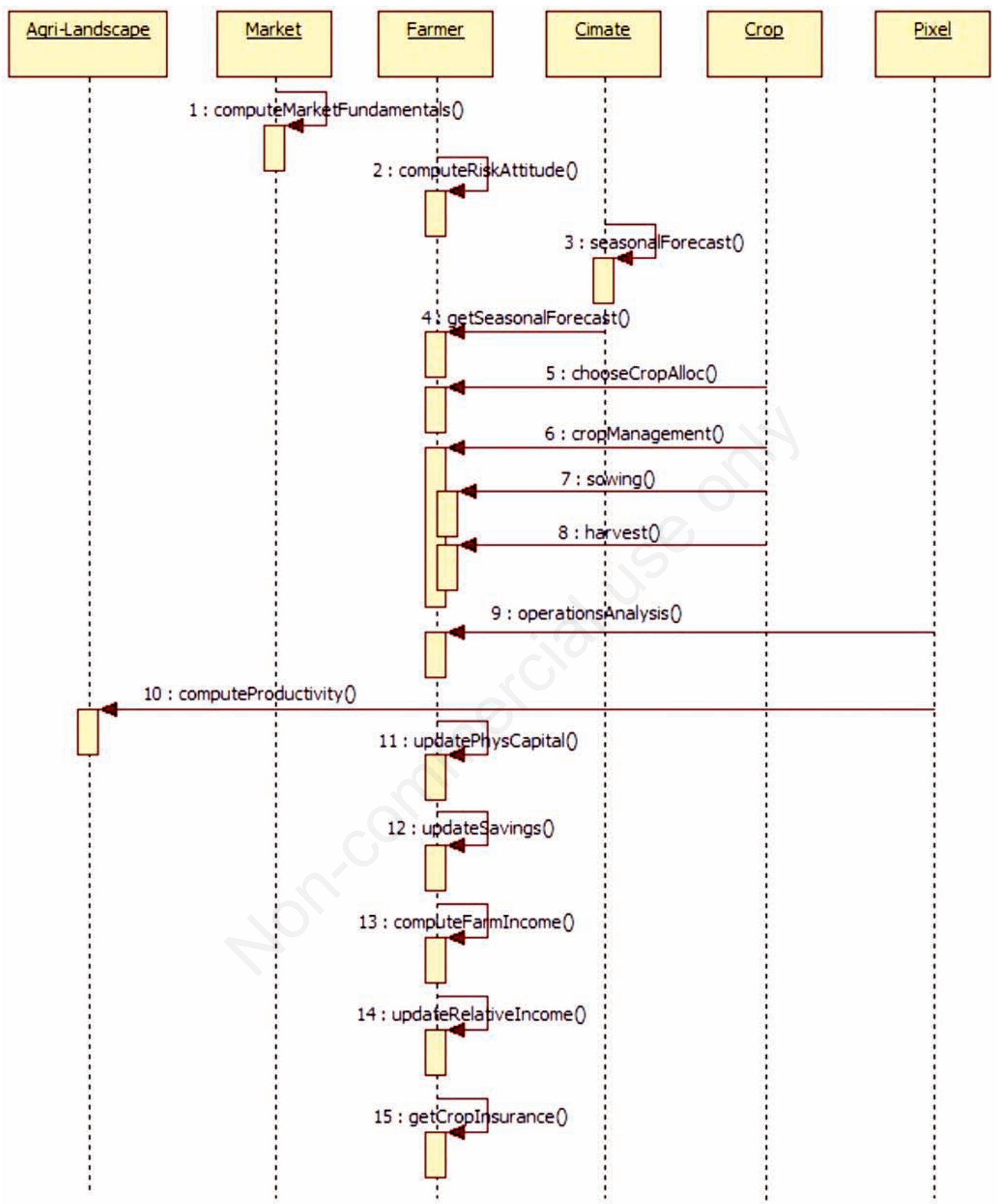

Figure 2. The Unified modelling language sequence diagram of strategic level. Operations are numbered according to their typical ordering during the running of one year. Arrows explain the flow of information for a certain operation to be performed by the receiving entity. 
nates]. It is, therefore, possible to compare future water demand and crop yield for the study area under different model configurations regarding: i) initialisation; ii) irrigation system in place; iii) climate service quality.

\section{Farmers' decision making}

The farmers' decision model largely depends on their classification in terms of risk attitude. In our model it is assumed that age and share of income (i.e. off-farm income) are the main variables that affect risk attitude (e.g. Moscardi and de Janvry, 1977). This characteristic of the farmer and the available information about weather forecast define the main farming choices.

In order to inform the farmers about future weather conditions, two types of meteorological services are made available: i) the bi-weekly agro-meteorological bulletin; and ii) the seasonal forecasts. The precise picture of future daily values of each meteorological variable pro- vided by the use of a synthetic climate record allows climate forecast services to be simulated. Bi-weekly bulletins and seasonal forecasting can, therefore, be produced by reading the record developed by the climate model for the future periods of interest. Varying levels of accuracy of the services are simulated by introducing random noise signals of various levels of magnitude within the procedure of reading the forthcoming values.

The bi-weekly bulletin provides the farmers with information about the cumulative precipitation forecast for the next three days, so that this amount is taken into account when defining the amount of water to be drawn for irrigation. The seasonal forecasts confirm any risk of drought in June and July, which is the most critical period for maize. As stated above, risk-averse farmers will tend to allocate more winter wheat in their UAA, while risk-taking farmers will decide to allocate more land to maize. This is captured in the UML activity diagram describing the crop allocation (Figure 3). Moreover, risk-averse farm-

chooseCropAlloc_ActivityDiagram
follows getSeasonalForecast 0 at the same timestep

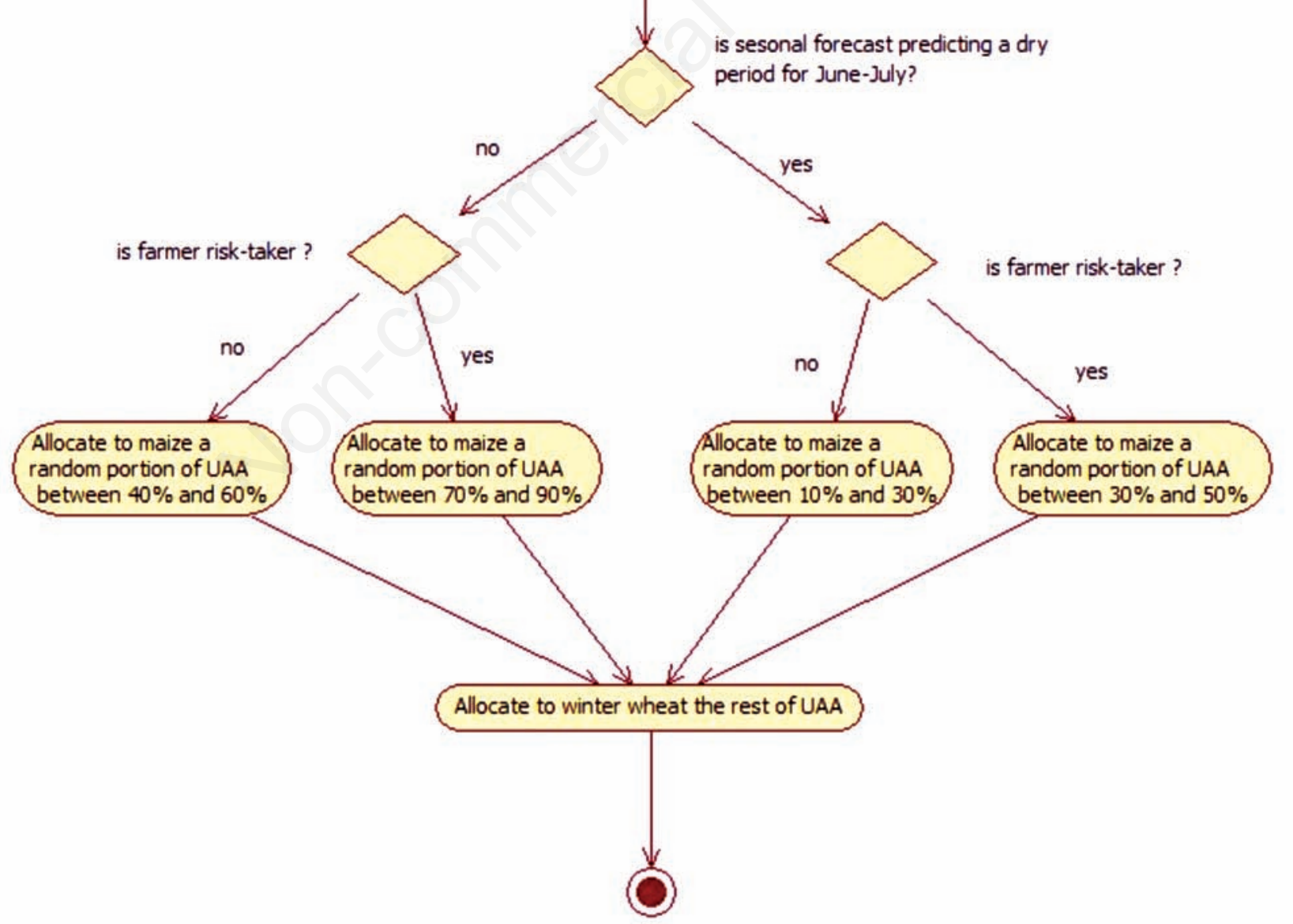

Figure 3. The UML activity diagram chooseCropAlloc. The operation is explained trough a flow diagram that is subject to two decision stages, captured by the diamond shapes. In order to decide the seasonal allocation of Utilised Agricultural Area to maize, a farmer is first informed about the seasonal forecasts and then the allocation decision is taken according to the assigned risk attitude. 
ers tend to save up money to buy an MPC-type insurance.

In the current prototype, no individual or collective learning is included in the decision process. However, it is planned to include memory about forecast quality and an individual learning process that can affect the farmer's choice to follow or not to follow the forecasts, depending on the effects of their past decisions.

There is no interaction among farmers, while each farmer interacts with his unitary land parcel, i.e. an extremely simplified farm, in which climatic and other records (soil, crop, irrigation system, and water infrastructure systems) are stored.

At the farm-level, there are various degrees of heterogeneity: 2 crops, 3 disjoint types of irrigation systems, 2 disjoint types of water infrastructure systems. Different elements of stochasticity are included to introduce variability in the model dynamics (Table 1).

The crop selling price and cost of production include a normally distributed stochastic factor to be applied to base values so that the parameters are different every year. The allocation of crops is made on the basis of random choices within fixed intervals designed to represent risk attitude (Figure 3). This also holds for the alpha $(\alpha)$ parameter that defines the share of profits to be re-invested rather than saved (Eq. 2). Both the initial age and the relative income of farmers are randomly distributed.

The off-farm salary and the living expenses affecting the farmer's total income inflow and outflow are based on random parameter values within fixed intervals.

The uncertainty of meteorological services is based on an error factor randomly distributed between its positive and negative value, to be set at initialisation.

\section{Overall structure of the model}

Figure 4 shows the main components of the model designed in UML and described in the previous section, although the current version of the prototype can still be considered as a toy model, i.e. a tool to be used to explore the main simulation issues before moving to a further coding phase for the full development of the original design. The core module (Farming practices) provides routines for simulating farming activities aimed at delivering a final product, i.e. a commercial commodity contributing to farmers' incomes. That module is further subdivided into five sub-modules providing the routines for: i) soil water balance, calculated according to the soil parameters, weather conditions and the cultivated crop; ii) water consumed by the crops; iii) use of irrigation water and scheduling according to crop needs and soil water conditions; iv) crop accumulation of biomass and final yields; $v$ ) profits deriving from fixed and variable costs (the latter being determined here only by irrigation method and water use), and from the yields multiplied by the market values of the commodities.

Besides the farming module in Figure 4, Crop physiology and Market is a module providing all the parameters needed to simulate the two selected crops (maize and winter wheat) and does not include dynamic variables. Similarly, the Soils module on top provides parameters for the calculation of soil water dynamic (in the current version only one soil has been implemented), while the Irrigation module gives relevant parameters for the identification of the irrigation system, in particular in terms of efficiency and costs. The Climate module contains the meteorological records produced by the climate model described

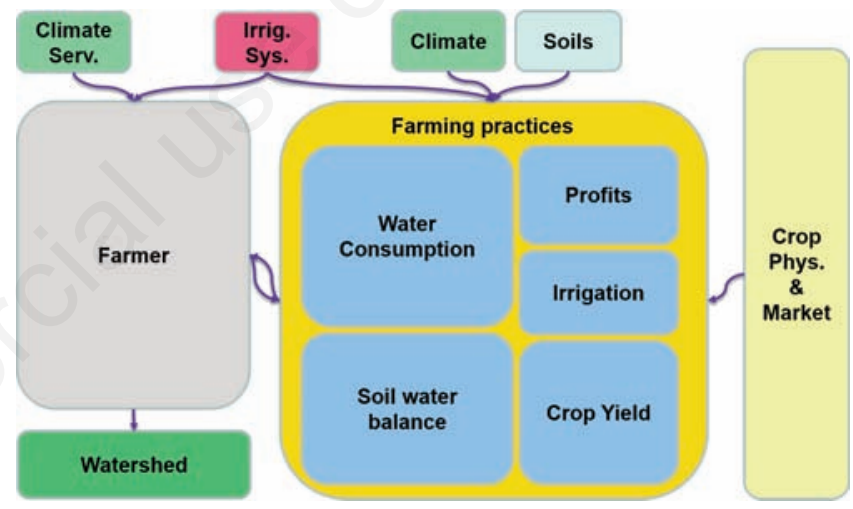

Figure 4. Modules of the Simile model and their main directional connections.

Table 1. Main behavioural parameters at initialization.

\begin{tabular}{|c|c|c|c|}
\hline Parameter & Value & Parameter & Value \\
\hline Number of farmers & 25 & Insurance premium & $10 \%$ guaranteed income* \\
\hline Age & Rand $^{\circ}(30,60)$ & Maize maximum yield & $13,000 \mathrm{~kg}$ ha- 1 \\
\hline Bank account & 0 & Wheat maximum yield & $8000 \mathrm{~kg} \mathrm{ha}^{-1}$ \\
\hline Maize allocation & $50 \%$ & Average maize production cost & $1500 € \mathrm{ha}^{-1}$ \\
\hline $\mathrm{K}$ & 1000 & Average wheat production cost & $1000 € \mathrm{ha}^{-1}$ \\
\hline Depreciation of $\mathrm{K}$ & $5 \%$ & Maize cost variability & $6.6 \%$ \\
\hline Farm income & Rand $^{\circ}(0,100)$ & Wheat cost variability & $2 \%$ \\
\hline Off-farm income & 100 - Farm Income & Average maize selling price & $200 € \mathrm{t}^{-1}$ \\
\hline For risk taker & $\operatorname{Rand}^{\circ}(0.5,1)$ & Average wheat selling price & $225 € \mathrm{t}^{-1}$ \\
\hline For non-risk-taker & Rand $^{\circ}(0,0.5)$ & Maize price variability & $10 \%$ \\
\hline Yearly off-farm salary & Rand $^{\circ}(100,500)$ & Wheat price variability & $4.4 \%$ \\
\hline Daily living expenses & Rand $^{\circ}(0.3,0.6)$ & Drought threshold for June-July & $<100 \mathrm{~mm}$ \\
\hline
\end{tabular}

*Guaranteed income is derived from the selling price of the guaranteed yield which is set at $50 \%$ of maximum yield; ${ }^{\circ} \mathrm{a}$ random number is picked within the interval. K, physical capital. 
below on a daily basis, while the Climate service stores the results of the elaborations in terms of forecasts and the uncertainty parameters used to explore the effects of degrading quality of the service in terms of bi-weekly and seasonal forecasts.

On the left of the model interface is the Farmer module in which the strategic and tactical decision-making procedures are provided in terms of: i) allocation of land to the two crops in consideration of the seasonal climatic forecast providing an estimate for June-July rainfall; and ii) irrigation management. The multiple agents act within this module. In other words, this module is replicated in the model to provide parallel simulations of a number of farmers with different age, risk attitude, income structure, etc. The last module, Watershed, is designed to store grid-based spatial information about farmers, soils, reference weather stations and irrigation infrastructures, and to produce spatially explicit and overall estimates of the main variables. In the current prototype, this module stores only the equations to calculate total values of the main variables.

More detailed visual presentation of the structure of the model and of its graphical interface is provided in Annex 1.

\section{Climate scenario}

Simulated climatic conditions are produced with the Consortium for Small-scale Modeling-Climate Mode (COSMO-CLM) model considering the IPCC A1B scenario (Scoccimarro et al., 2011). COSMO-CLM is based on the operational non-hydrostatic mesoscale weather forecast model developed by the German Weather Service. This model has been used within the framework of the FUME EU project (http://www.fumeproject.eu/) to generate a set of climate projections covering the period 1970-2100. In the version implemented over the EuroMediterranean region, the horizontal resolution is $0.12^{\circ}$ (approx. 14 $\mathrm{km})$. For the purpose of this study, we considered the daily precipitation over the period 2015-2030 simulated for 5844 daily time steps.

The input data used are those of the climate station of Legnaro (PD), which is centrally located within the VLW. In the prototype model, a forecasted cumulative precipitation below $100 \mathrm{~mm}$ for the months of June and July is considered as drought index signal for the farmers (Figure 5).

\section{Economic scenario and risk-taking attitudes}

Risk attitude is computed by means of a bi-dimensional fuzzy membership function (Kelly and Painter, 1996) that assumes two hypothetical farmers: i) a 20 -year old farmer(j) producing $0 \%$ of his income from farming activities representing the perfect risk taker (i.e. $\mathrm{P}=+1$ ); and ii) a 90 -year old farmer(i) with $100 \%$ of his income from farming activities representing the perfect risk-averse farmer (i.e. $\mathrm{P}=-1$ ). Other farmers are compared to these two extremes such that a farmer is considered risk-taker for $\mathrm{P}>0.1$, or risk-averse, according to the following function:

$$
\rho_{i \mid j}(\Lambda)=\frac{\left|\vec{v}_{i}\right|^{2}-\left|\vec{v}_{j}\right|^{2}}{\left|\vec{v}_{i j}\right|^{2}}
$$

where:

$\Lambda$ is the farmer for whom we want to compute the risk attitude, identified by a certain age and share of income.

$\bar{v}_{i}$ is the vector from $\Lambda$ to farmer(i);

$\bar{v}_{j}$ is the vector from $\Lambda$ to farmer(j); and

$\bar{v}_{i j}$ is the vector from farmer(i) to farmer(j).

In accordance with Hasselmann and Kovalevsky (2013), economic growth is made to be dependent on the way businesses choose to invest their own profits. Following this logic, the economics of farmers is considered dynamic throughout the simulation, and is based on the evolution of three key variables: physical capital, savings and insurance (premium and indemnity). Physical capital $(\mathrm{K})$ represents the physical assets of the farmer including rural buildings, machines and irrigation systems. The variation of physical capital (i.e. updatePhysCapital operation) is given by the function:

$$
\Delta K=i k-\lambda k \cdot K
$$

where:

$i k$ is $\alpha \cdot$ profits, in which $\alpha$ represents the share of profits re-invested in $\mathrm{K}$, and $\lambda \mathrm{k}$ is the depreciation rate of $\mathrm{K}$.

Similarly, the savings (S) represent the bank account of the farmer for his farming activities. The variation in savings (i.e. updateSavings operation) is given by the function:

$$
\Delta S=i s-\lambda s \cdot S
$$

where:

is is $\beta$. profits; and $\beta$ is the appreciation rate of $\mathrm{S}$.

Savings allow the farmer to allocate monetary resources for insurance practices. In particular, we assume that, if farmers have enough savings to pay to insure their yield, then they decided to pay the insurance premium. This will in turn reduce the total savings available for the future but it will make the farmer eligible for an indemnity if the yield is below $50 \%$ of that expected. Furthermore, every farmer has a certain flow of off-farm income and living expenses which are randomly assigned.

\section{Simulation scenario}

The model is initialised with 25 farmers and age is randomly assigned between 30 and 60 years. During the simulation, when the farmers reach the age of 65 they are substituted by younger farmers,

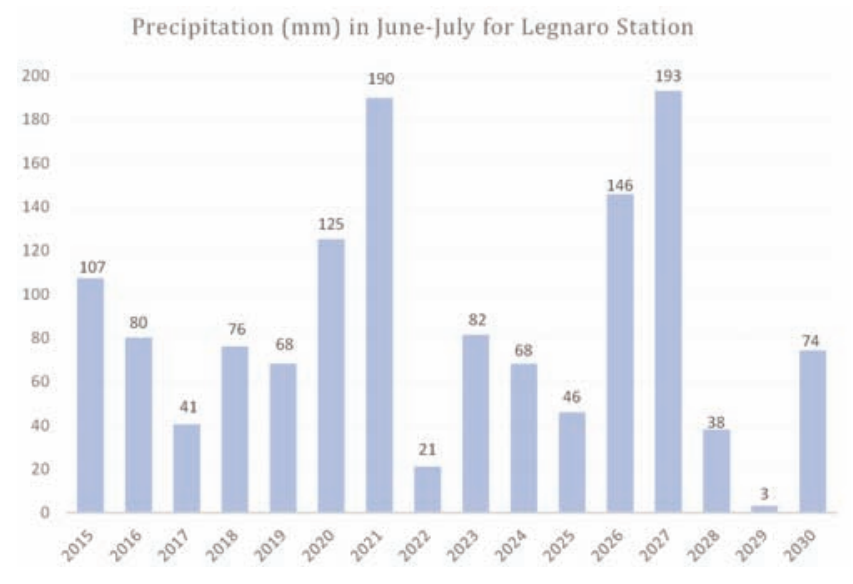

Figure 5. Precipitation input data for June-July 2015-2030 (A1B scenario). 
simulating a second generation. The share of income from farming is randomly distributed between $0 \%$ and $100 \%$, to represent different categories of farms, from hobby activities, to part-time farming and full-time professional activities. The irrigation system in place and the accuracy of the meteorological services are exogenous scenarios to be selected at initialisation. Table 1 shows the values of the main behavioural parameters at initialisation related to the farmer and the farming activities. The parameters, particularly those expressed as point values, have been set for exploratory purposes on the basis of the Authors' experience supported by available average reference values (INEA, 2007).

\section{Results and discussion}

Figure $6 \mathrm{~A}$ shows the ageing of each farmer with a stepping line and how the stochastic component of the model applied to the group of farmers simulated in parallel determines varying initial ages of farmers, who eventually leave their farms to descendents (aged around 35 years) when they reach the age of 65 . As a consequence of varying farmers' age and attitude, the same signal, i.e. a seasonal forecast, produces varying outcomes of the farmer decision sub-model in terms of crop allocation. Figure 6B shows histograms of relative land allocation to maize (i.e. percentage of total UAA) as determined by the seasonal forecasts and the varying risk attitude of the same cohort of farmers reported in Figure 6A. The allocation of land follows the real variability of summer rainfall (Figure 5), as farmers are informed about the forthcoming summer rainfall by the climatic services providing seasonal forecasts. As stated above, given the use of a predefined synthetic weather forecast, generated by the climatic model, the forthcoming seasonal precipitations are known with certainty, but their values were degraded in consideration of forecast uncertainty by including a random noise signal altering the values within a maximum range of $+/-30 \%$.

Given the several sources of stochasticity included in the model, many simulation runs are needed to explore model results and identify what could be the effects of the different scenario variables.

Figure 7 shows the distribution of results of nine sets of 100 simulations run in parallel on cohorts of 25 farmers, aimed at exploring the conjoint effects of alternative irrigation systems with varying quality of the climatic forecast services on overall water withdrawals and farmers' incomes (The Y scale has been deliberately made unreadable to stress the fact that the model has to be considered as an explorative tool which has not yet reached a consolidation step, allowing discussion of the details of outputs in absolute terms.). The parameters representing three irrigation systems (gravity, sprinkler and drip irrigation) were subsequently set at the launch of simulations, with a factorial combination with three levels of forecast uncertainty, to represent possible levels of quality of climate services: random noises ranging within the extremes of $+/-10 \%, 30 \%$ and $90 \%$ (L, $\mathrm{M}$ and $\mathrm{H}$ uncertainty, respectively), applied to the weather-generated precipitation values communicated to farmers.

The results show that the effect of irrigation systems (Figure 7A) is more evident in terms of water volumes than for farmers' incomes (Figure 7B). The reason lies in the levels of variable costs that have been adopted for the irrigation systems: higher variable costs were given for the more efficient irrigation systems. Again the values adopted for variable costs were defined for exploratory purposes and require accurate consideration in case of future operational use of the model, considering, for example, differentiated costs for the different crops, with distinction of seasonal costs (e.g. setting up the irrigation devices in the field) from costs related to the functioning of the irrigation system (e.g. cost of energy for pumps). In spite of the level of first approximation of model set up, Figure 7 shows how bad forecasts (high value of random noises) affect the performance of irrigation systems (Figure 7A) with much wider whiskers (spread of the distribution curves of water withdrawals) for the $\mathrm{H}$ uncertainty levels, and in particular for the systems with lower efficiency. Even more significantly, bad quality of climate services reduces farmers' incomes (Figure 7B) thus representing the cases in which they are penalised because they trusted bad forecasts and encountered unexpected weather (e.g. drought with a lot of maize cultivated in the farm), thus having to afford high costs for irrigation. In the case of incomes, the graph boxes (including the central portion of $50 \%$ of the model outputs) do not show evident differences in terms of performances of the three irrigation systems (the vertical ranges of the boxes are similar). However, they do show the interesting feature of much greater instability of incomes, in particular in the case of sprinkler irrigation (high energy expenses) and gravity (very low efficiency and consequent very high water withdrawals).

One consequence of the observed behaviour of the simulated system is that one should expect remarkable changes in the attitudes of farmers towards climatic services over time, depending on the consequences of considering such information in the light of past decisions: for example, in the case of initial low quality services provided to farmers, a permanent negative attitude about their use in decision making should be expected. Moreover, it should be expected that mutual learning processes should be in place between farmers, determining significant phenomena of propagation of experiences and attitudes. Both these two dimension of the problems are considered in the UML design of the model, but not yet implemented in Simile.

\section{Conclusions}

Mainstream economic models are usually not capable of analysing the emergent properties developing within heterogeneous and spatially distributed social-ecological systems, because they prefer to focus on average and aggregated agents and compartments, and they adopt the assumptions of neoclassical economics, such as fully rational and informed economic agents. These limitations in turn affect many recent research efforts aimed at providing integrated assessment models for climate change adaptation, with the effect of missing in particular the possibility of analysing autonomous adaptation processes as implemented by multitudes of agents (e.g. farmers), in response to various drivers and stimuli, such as the evolution of markets and relevant policies and legislation.

Similarly, agronomic models usually adopt deterministic approaches, in particular when integrated into agro-economic models that typically adopt optimisation routines (e.g. linear programming) and, again, they tend to represent idealised rational farmers behaving either simply as pure profit optimisers or, in some cases, in some way also integrating risk-taking functions.

The observation of autonomous adaptation processes demonstrates that bounded rationalities, imperfect information, varied utility functions, together with the effects of spatial topology and communication networks have a remarkable effect on the overall behaviour of the system. Dealing with these issues requires a new generation of integrated models in which the human dimension is carefully considered together with accurate simulation of the physical and environmental dimensions (Giupponi et al., 2013).

The present work attempts to go beyond consolidated approaches by including consideration of various sources of variability and uncertainty for the analysis of farm management and water uses for irriga- 
tion in a stylised agricultural landscape, formalised with data from the VLW, an area in which climate change is projected to have significant effects on climatic variables in the future thus determining the need for effective adaptation strategies.

One planned CCA strategy often mentioned is improved farm management thanks to innovative climatic information services, such as accurate short-term weather forecasts (2-5 days) and seasonal forecasts, although the effectiveness of such services is still debated (Bergez and Garcia, 2010).

The results presented here provide a preliminary idea about how the agricultural system can behave and react to both climatic trends and climate services, with a focus on farmers' incomes and water use for irrigation. The prototype model illustrates that bad forecasts may significantly affect farmers' incomes, in particular in those cases in which irrigation systems are characterised by high variable costs (e.g. energy required for irrigation pumps). It also shows how the interaction between various sources of stochasticity and uncertainty may affect aggregated and average results.

Very importantly, the results obtained demonstrate the possibility of exploring jointly the interactions between autonomous (farmers' decisions) and planned (climate services) adaptation.

As the model has been applied for explorative purposes, there are several limitations and assumptions that must be considered when deriving specific conclusions from the results. Not all the functionalities foreseen in the UML diagram have been implemented in this prototype model. In the current version of the model, no individual or collective learning is included in the decision-making process, as interaction among farmers and the effects of penalisation from bad decisions are not yet considered. The model presented here is, therefore, more similar to a micro-simulation model than to a fully devel-
A

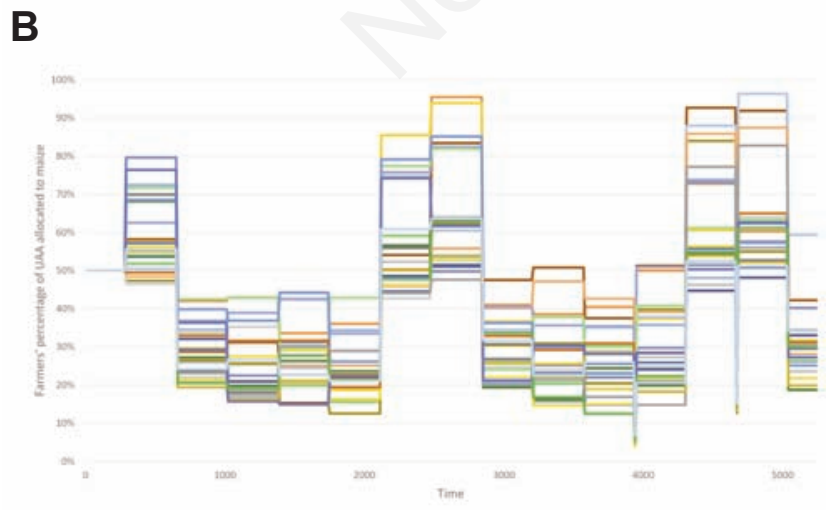

Figure 6. Simulation runs for 15 years with a cohort of 25 farmers in parallel. Ages of farmers, leaving farms to descendents around the age of 65 (A), and effects of seasonal forecasts and risk-taking attitude in determining the allocation of land to maize crop (B).
A

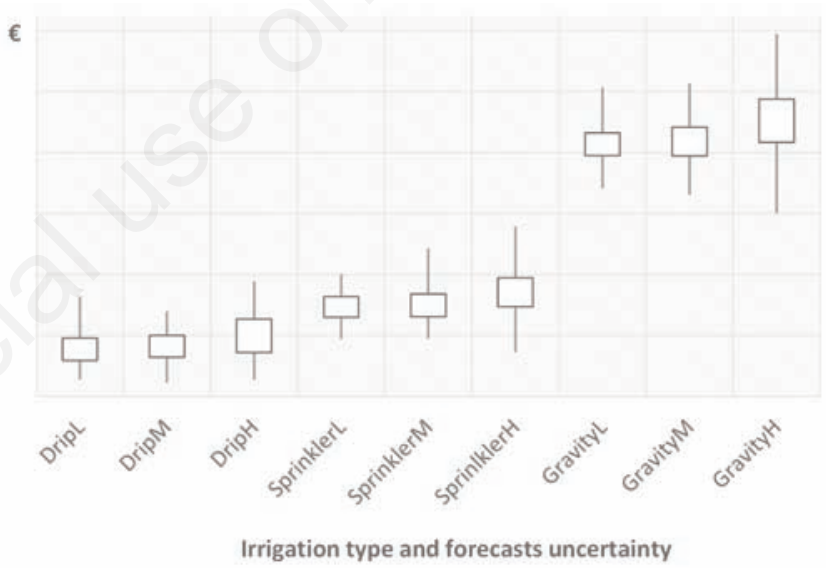

B

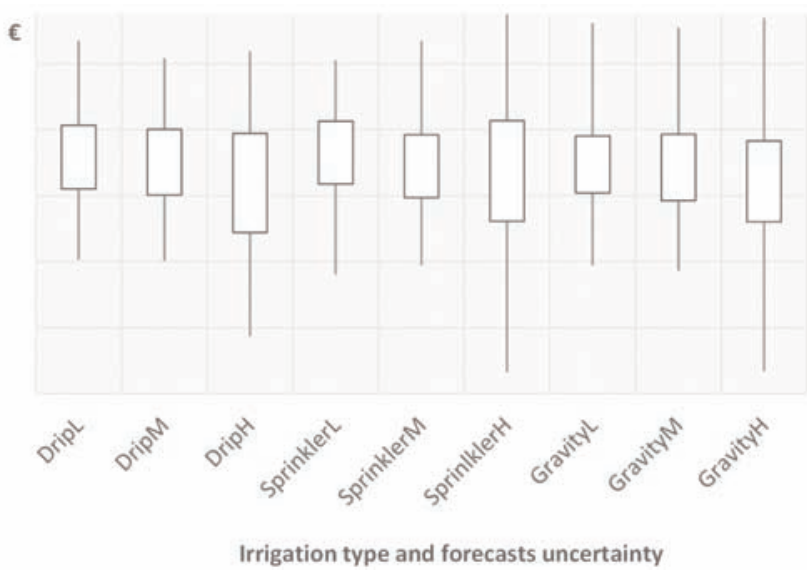

Figure 7. Box and whisker plots of irrigation volumes (A) and farmers' incomes $(B)$ as resulting from nine sets of 100 simulation for drip, sprinkler and gravity irrigation systems, and with varying levels of uncertainty of the seasonal forecasts introduced by altering the June-July rainfall with random noises ranging within the extremes of $+/-10 \%(\mathrm{~L}) ; 30 \%(\mathrm{M})$ and $90 \%(\mathrm{H})$. 
oped agent-based model, as described in Troitzsch (2013). Moreover, explicit consideration of spatial variability of model parameters is not included in the current version. In addition, we investigate only a single climate scenario, the IPCC A1B scenario.

Most of the above-mentioned limitations were already considered in the UML design but have not yet been implemented in the Simile modules. Further developments are required not only concerning the consolidation of the model parameters and validation, but also in the expansion of the important parameters, e.g. learning and mutual learning, in order to simulate complex social-ecological systems. Improved analysis can be achieved through incorporating heterogeneous soil types and multiple climate scenarios. For spatially explicit outcomes, the model needs to be integrated with GIS.

These developments will increase the complexity of the modelling tool by orders of magnitude. Parallel to these, adequate plans and instruments will be needed to assess the overall quality of simulation outcomes. It, therefore, seems to be extremely important to: i) continue the development according to a robust and conceptually sound modular structure; ii) provide the capabilities for the analysis of sensitivity, calibration, and validation of each module in isolation from the other and in combination with them; iii) develop adequate procedures for the analysis of uncertainty and error propagation.

As far as the environment for model development is concerned, Simile, has been proved to be very powerful and efficient for the coding of the UML design and for facilitating collaborative work. However, at the same time, it appeared to be not so well suited for managing the level of complexity required by the full development of the UML design into a multi-agent spatial model, with consideration of multiple sources of uncertainty and stochasticity. The complexity of the prototype is clearly shown in Annex 1, even in its preliminary simplified version. Agent-based modelling suites might prove to be more efficient, but at the price of encountering new problems in representing the water balance dynamics and spatial features. However, even if in future developments the Simile environment were to be abandoned, the work done in the UML design would allow a non-ambiguous and facilitated migration to other modelling environments.

\section{References}

Allen RG, Pereira LS, Raes D, Smith M, 1998. Crop evapotranspiration: guidelines for computing crop water requirements. FA0 Irrigation and Drainage Paper, No. 56. United Nations, Food and Agriculture Organization, Rome, Italy.

Balbi S, Giupponi C, Perez P, Alberti M, 2013. A spatial agent-based model for assessing strategies of adaptation to climate and tourism demand changes in an alpine tourism destination. Environ. Model. Softw. 45:29-51.

Bhandari S, 2011. Modeling future irrigation water demand in the Venice Lagoon Watershed: A system dynamics approach. Master's thesis, Department of Physical Geography and Quaternary Geology, Stockholm University, Stockholm, Sweden.

Bharwani S, Bithell M, Downing TE, New M, Washington R, Ziervogel G, 2005. Multi-agent modelling of climate outlooks and food security on a community garden scheme in Limpopo, South Africa. Philos. T. Roy. Soc. B. 360:2183-94.

Berger T, 2001. Agent-based spatial models applied to agriculture: a simulation tool for technology diffusion, resource use changes and policy analysis. Agr. Econ. 25:245-60.

Bergez JE, Garcia F, 2010. Is it worth using short-term weather forecasts for irrigation management? Eur. J. Agron. 33:175-81.

Bojovic D, Bonzanigo L, Giupponi C, 2012. Drivers of change in
Southern European agriculture: online participatory approaches for the analysis of planned and autonomous adaptation strategies. In: R. Seppelt, A.A. Voinov, S. Lange, D. Bankamp (eds.) Managing resources of a limited planet: pathways and visions under uncertainty. International Environmental Modelling and Software Society, Leipzig, Germany, pp 684-91.

Cossarini G, Libralato S, Salon S, Gao X, Giorgi F, Solidoro C, 2008. Downscaling experiment for the Venice lagoon. II. Effects of changes in precipitation on biogeochemical properties. Clim. Res. 38:43-59.

Dutton JA, 2002. Opportunities and priorities in a new era for weather and climate services. B. Am. Meteorol. Soc. 83:1303-11.

Giupponi C, Azzellino A, Salvetti R, Parati P, Carpani M, 2012. Water quality assessment in the Venice Lagoon Watershed with multiple modelling approaches. In: R. Seppelt, A.A. Voinov, S. Lange, D. Bankamp (eds.) Managing resources of a limited planet: pathways and visions under uncertainty. International Environmental Modelling and Software Society, Leipzig, Germany, pp 1327-34.

Giupponi C, Borsuk ME, de Vries BJM, Hasselmann K, 2013. Innovative approaches to integrated global change modelling. Environ. Model. Softw. 44:1-9.

Hasselmann K, Kovalevsky D, 2013. Simulating animal spirits in actor-based environmental models. Environ. Model. Softw. 44:10-24.

INEA (Istituto Nazionale di Economia Agraria), 2007. Database of Rete d'Informazione Contabile Agricola (RICA). Available from: http://www.rica.inea.it/public/it/accesso_dati.php

INEA (Istituto Nazionale di Economia Agraria), 2009. Rapporto sullo stato dell'irrigazione in Veneto a cura di Raffaella Zucaro e Andrea Povellato. INEA (Istituto Nazionale di Economia Agraria), Roma, Italy. Available from: http://www.inea.it/ap/ bollettini/docs/Irrigaz_VenetoWeb.pdf

ISMEA (Istituto di Servizi per il mercato agricolo e alimentare), 2012. Cereals price database. Available from: http://www. ismea.it/flex/ cm/pages/ ServeBLOB.php/L/IT/IDPagina/188

Iturrioz R, 2009. Agricultural Insurance. Primer series on insurance, issue 12, November 2009. The International Bank for Reconstruction and Development/The World Bank, Washington, DC, USA. Available from: http://siteresources.worldbank.org/ FINANCIALSECTOR/Resources/Primer12_Agricultural_Insuranc e.pdf

Kelly W, Painter J, 1996. Hypertrapezoidal fuzzy membership functions. pp 1279-1284 in 5th IEEE Int. Conf. Fuzzy Systems, New Orleans, LA, USA.

Moscardi E, de Janvry A, 1977. Attitudes toward risk among peasants: an econometric approach. Am. J. Agr. Econ. 59:710-16.

Müller B, Bohn F, Dreßler G, Groeneveld J, Klassert C, Martin R, Schlüter M, Schulze J, Weise H, Schwarz N, 2013. Describing human decisions in agent-based models - ODD+D an extension of the ODD protocol. Environ. Model. Softw. 48:37-48.

Salon S, Cossarini G, Libralato S, Gao X, Solidoro C, Giorgi F, 2008. Downscaling experiment for the Venice lagoon. I. Validation of the present-day precipitation climatology. Clim. Res. 38:31-41.

Scoccimarro E, Gualdi S, Sanna A, Bucchignani E, Montesarchio M, 2011. Extreme events in high resolution CMCC regional and global climate models. CMCC Research Paper, RP0110, 2011. Centro Euro-Mediterraneo sui Cambiamenti Climatici (CMCC), Lecce, Italy. Available from: http://www.cmcc.it/publications/ rp0110-extreme-events-in-high-resolution-cmcc-regional-andglobal-climate-models

Smit B, Pilifosova 0,2001. Adaptation to climate change in the context of sustainable development and equity. In: J.J. McCarthy, 
O.F. Canziani, N.A. Leary, D.J. Dokken and K.S. White (eds.) Climate change 2001: impacts, adaptation and vulnerability. Contribution of Working Group II to the Third Assessment report of the Intergovernmental Panel on Climate Change. Cambridge University Press, Cambridge, UK, pp 877-912.

Smit B, Skinner MW, 2002. Adaptation options in agriculture to climate change: a typology. Mitig. Adapt. Strateg. Global Change 7:85-114.
Troitzsch KG, 2013. Historical introduction. In: B. Edmonds and R. Meyer (eds.) Simulating social complexity. Springer, BerlinHeidelberg, pp 13-21.

Ziervogel G, Bithell M, Washington R, Downing T, 2005. Agent-based social simulation: a method for assessing the impact of seasonal climate forecast applications among smallholder farmers. Agr. Syst. 83:1-26. 